\title{
Impact on clinical learning during coronavirus disease 2019 pandemic: from disruption to recovery and the new norms
}

\author{
Yi Xiang Tay ${ }^{1}$, Hwei Chuin Chow ${ }^{1}$ and Chin Chin Ooi ${ }^{1,2}$ \\ ${ }^{1}$ Division of Radiological Sciences, Radiography Department, Singapore General Hospital and ${ }^{2}$ Health and Social \\ Sciences, Singapore Institute of Technology, Singapore
}

\section{Introduction}

Singapore's first novel coronavirus disease 2019 (COVID-19) case surfaced on January 23, 2020 and has since risen past 57,000 cases at point of writing. The pandemic has brought unprecedented education disruption. With a near-total closure of academic institutions and lockdown of clinical sites, many students' clinical teaching was postponed. It is imperative to implement contingency measures to make up for the lost learning opportunity while ensuring learning continues in a safe environment. In this article, we focus on narrating our experiences as radiography clinical educators in sustaining students' education, our responses to the pandemic and insights to future norms of clinical education at a large tertiary referral hospital in Singapore.

\section{Disruption of clinical placement}

Following the experiences from severe acute respiratory syndrome (SARS), Singapore had introduced the Disease Outbreak Response System Condition (DORSCON) framework in 2006 to guide responses according to the severity of a pandemic [1]. By end of January 2020, the hospitals had suspended postings for all international students. In the span of a week, local students followed suit after the government's decision to raise the DORSCON level from Yellow to Orange. These scenes had brought back a sense of déjà vu a reminder of the 2003 SARS outbreak.

\section{Resumption of clinical placement}

With transition of risk assessment level to DORSCON Orange, both clinical trainings and clinical assessments
Received: June 25, 2020 • Revised: September 13, 2020 • Accepted: October 15, 2020 Corresponding Author: Yi Xiang Tay (https://orcid.org/0000-0001-8322-1500) Department of Diagnostic Radiology, Singapore General Hospital, Block 2 Level 1, Outram Road, Singapore 169608

Tel: +65.91270879 Fax: +65.62433126 email: Tay.yi.xiang@sgh.com.sg
Korean J Med Educ 2020 Dec; 32(4): 297-305.

https://doi.org/10.3946/kjme.2020.176

eISSN: 2005-7288

(C) The Korean Society of Medical Education. All rights reserved. This is an open-access article distributed under the terms of the Creative Commons Attribution Non-Commercial License (http:// creativecommons.org/licenses/by-nc/3.0/), which permits unrestricted non-commercial use, distribution, and reproduction in any medium, provided the original work is properly cited. 
were postponed. Some higher education institutes (HEIs) had their student term breaks brought forward while others explored clinical assessments in the form of simulated or hybrid simulations [2]. However, by early February 2020, there were growing concerns from all stakeholders on the educational and manpower impact of delayed clinical training for allied health professionals. There was a need to prepare a contingency plan for students' clinical training and ensure the sustainability of learning during such crisis. Fortunately, with the collective effort of the HEIs and hospitals, approval was granted by Singapore Ministry of Health: clinical training could resume in phases - starting with the penultimate year students. Coordinated response from the HEIs and hospitals were required for this group of "COVID-19 generation" students who must continue their education in an environment transformed by physical distancing and heavily influenced by infection control measures.

\section{Safe management in the HEls and hospitals}

In any pandemic outbreak, the main responsibility lies with the HEIs and hospitals to ensure the safety of staff, patients, and students [3]. Following a rapid increase in the number of confirmed cases, precautionary measures were implemented to safeguard the safety of these stakeholders. Educators were instituted to monitor students' compliance to the hospital measures practice of good hand hygiene and donning of surgical masks when in close contact with high risk or immunocompromised patients.

Significant leg work was also done at the background by the HEIs to ensure that compliance with the safety protocols were in place. Similar to the Australian medical schools, local HEIs instigated online refresher training for students on hand hygiene, proper donning and doffing of personal protective equipment (PPE) [4]. This was unlike the previous training which was embedded within their usual curriculum. A pre-clinical briefing was conducted to educate the students on the

Fig. 1. Clinical Placement Risk Mitigation Measures

\section{Risk mitigation measures}

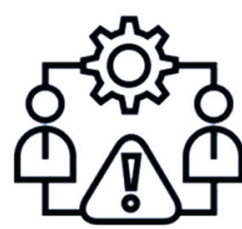

- Students are to be trained in small groups

$\checkmark$ Clinical educator to student ratio (1:1)

- Avoid high risk area, procedures, and patients

- AGPs are avoided

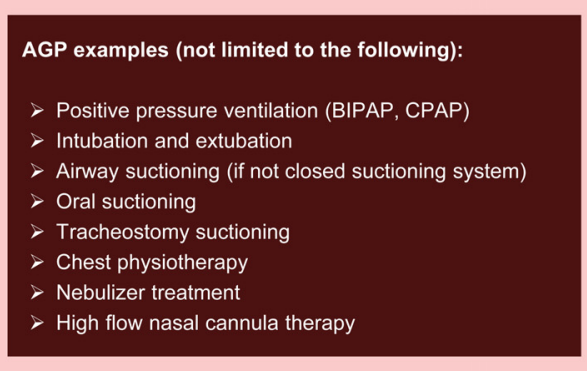

Naso-pharyngeal/oral pharyngeal swab collection

Sputum induction

Endoscopy (including bronchoscopy)

$>\mathrm{CPR}$

$>$ Manual ventilation (air-viva bagging) before intubation

$>$ High speed devices use for surgical procedures

$>$ Dental

$>$ Tracheostomy creation/insertion

$>$ Nasogastric tube insertion

AGP: Aerosol generating procedures, CPR: Cardiopulmonary resuscitation. 
curated guidelines for clinical education in the COVID-19 era and to address any concerns that they might have for the upcoming clinical training. In addition, compliance to risk control measures was emphasized and documented in the form of a student clinical practice information package [5]. We coordinated with the administrative staff and established new protocols such as daily twice temperature monitoring, students' rostering, and sick management as well as risk mitigation measures (Fig. 1). Stringent precautionary measures and guidelines for safe clinical training were developed as part of the orientation program. An email was sent and addressed to all clinical educators to update on the clinical training and highlight the risk control measures implemented. The communique detailed risk control measures -appropriate infection prevention and control measures, safe distancing, designated areas for meals, and donning of surgical masks at all times in clinical and public fronting areas were highlighted (Fig. 2). This was well received by all educators who were geared up to resume clinical teaching of students amidst the pandemic.

\section{Cohorting and controlled movements}

To align with local infection control measures, modification of clinical training was necessary, encouraging present curriculum to continue with minimal disruption [6]. Students were excluded from handling patients in high risk areas such as emergency department and intensive care units, to mitigate the risk of infection. It was a challenging decision made with considerations such as determining the appropriateness of having students working with COVID-19 positive patients [7]. While we have a reassuring availability of PPE in Singapore, there was a need to ration these limited resources in preparation for a prolonged pandemic battle, and hence the deliberate decision [8].

Peace time practices such as larger student groups, frequent movements between different healthcare institutions, and longer placement duration had been replaced with learning in shorter, smaller, and stable social bubbles -aligned to practices in the United Kingdom, Australia, and North America [9]. The students' health was of utmost priority in such decisions the presence of students had clearly been identified as an unnecessary potential vector of virus spreading.

Fig. 2. Institutional Safety Measures for Clinical Placement

\section{Institutional safety measures}

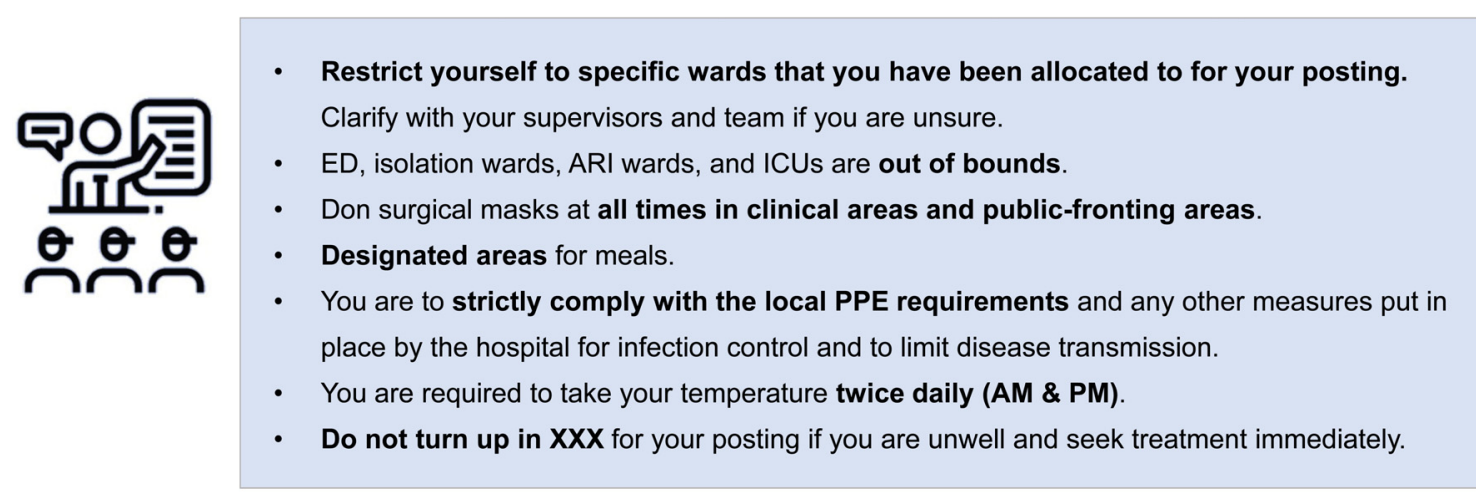

ED: Emergency department, ARI: Acute respiratory infection, ICU: Intensive care unit, PPE: Personal protective equipment. 
Clearly, this should be avoided.

As students were involved in general radiography where work was performed in a smaller size X-ray examination room, it was essential to prevent congregation. To facilitate safe physical distancing, only one student was allowed in each examination room -aligned with the requirements for safe management measures at the workplace [10]. We rostered students to be trained in small groups whereby there was an educator to student ratio of $1: 1$, from the previous ratio of $1: 2$. The division of the cohort into two separate groups facilitated the ratio of 1:1 [5]. The faculty numbers were further augmented by the cancellation, postponement of large group educational activities (conference, workshop, and seminars) and suspension of patient fronting research activities freed up more educators to support clinical training. Moreover, this aided in managing the potential increase in educators' burden due to the reduced educator-student ratio. Of note, educators also highlighted that the reduced ratio had enabled them to concentrate on teaching the assigned student, without having the burden of managing multiple students with different learning abilities in a clinical environment.

\section{Deployment of technology tools}

In tandem, we had to adapt swiftly to keep pace with new developments taking place nationally and across the world. For example, the pandemic had pushed us to embrace the digital world and the digital trajectory will only accelerate. In response to COVID-19, many precautions were taken, and modification of practices were made while leveraging the advancements in technology video conferencing and tele-supervision. To adhere to the preventative measure of physical distancing, the usual weekly radiographers face-to-face continuing professional development (CPD) sessions which the students usually attended had since transited to a video conferencing platform-Zoom. The continuum of these sessions and frequent educational webinars provided additional learning and assisted in the deficits arising from the current clinical environment and cancellation of physical tutorials. Fortunately, the pandemic had accelerated the widespread adoption of online teleconferencing in Singapore. All students had experience with Zoom during their home-based learning at the onset of the outbreak and similarly, radiographers had been leveraging the platform for their CPD needs. While tele-supervision was an option for supervision of local allied health professionals, it was not currently viable in radiography due to infrastructure, security concern, and patient conservatism tradition of Confucianism in our Asian cultural context [11]. With the new educator to student ratio, one could not be blamed to think that students would have better student-centric education in this "ideal" clinical supervision model. Assigned trained educators might be deployed to support various COVID-19 sites and they might be issued compulsory 5 days medical leaves if any presented with symptoms of acute respiratory infection. Students would then be assigned to be supervised by any available practicing radiographers of varying teaching experience. Such unavoidable circumstances could lead to a loss of learning opportunity. Fortunately for student radiographers, there were successful cases of implementing tele-supervision overseas. With globalization and cultural changes, there might be potential of adopting video conference technology in supervision locally. Students could benefit from technology to maximize learning through improved delivery of education [12], while ensuring standardized and quality supervision. 


\section{Faculty and students}

Learning contracts were in place prior to COVID-19 outbreak and most students were familiar with this approach -reducing time for students to be engaged. The collaborative development of the contract between student and educator through discussion and negotiation in developing of clinical competence is the keystone of successful learning. This is a golden opportunity for students in this challenging period to take ownership over their learning at the outset of placement, with support from their educator, creating an engaging learning environment. On the other hand, adequately trained educators are essential in providing guidance and encouragement during the contract development [13]. Trained educators can be scarce in clinical departments where manpower is stretched due to segregated roster and proactive reduction of backlog cases reopening after COVID-19 shutdown. To navigate this challenge, we sought the support and guidance from our radiography department management. Educators were excluded from the main operational roster to ensure that clinical training was not compromised due to the lack of faculty. Ground radiographers were updated on the resumption of student's placement to seek their understanding of having lesser manpower on the ground with the educator (colleague) guiding the student assigned in the same $\mathrm{X}$-ray examination roombalancing workload and clinical training.

Educators play a vital role in students' learning experience and ensure a safe environment that is welcoming, respectful, inclusive, and supportive, while continuing their efforts to prioritize the psychological well-being of their students. Continual change in workflow of clinical areas, engagement with new educators coupled with significant amount of knowledge and competency of practical skills required, can place significant pressures on students, and needs to be managed appropriately [14]. Moreover, student radiographers arriving for clinical training during a pandemic was not without associated stresses. They experienced fear and anxiety about their health and were worried about contacting COVID-19 while on placement [15]. With that in mind, we created a group in WhatsApp Messenger to communicate, stay connected, and share information with our students. Rapid communication with the students was established and incorrect information was minimized regular update of students through dissemination of latest daily routine instructions from the hospital's command center.

One should also not neglect social support while maintaining physical distance [14]. Strong relationship can still be built without compromising social connection and emotional closeness frequent follow-up of learning progress and engaging students or simply just being there. This played a crucial role when a radiographer was reported to have contracted COVID-19 while supporting a community facility. Words of reassurance and support were disseminated in the messenger group to establish that the department was a safe and supportive environment for learning. Regular check-ins with students were performed-prioritizing relationships.

Although the faculty is a first point of contact for students in distress, the latter need to learn and manage their stress and anxiety. Emphasis of the importance of self-care and healthy coping strategies were provided by the hospital to tide through this stressful period. Appreciation gift packs and thank you notes received from donors, public and the hospital management also went a long way in supporting the faculty who were balancing the role of an educator and a frontline healthcare worker. Through this form of interconnectedness, it created more purpose to their days and 
well-being in this challenging time [16].

\section{HEls and hospitals}

It is evident that a partnership approach towards adopting new practices-embracing new technology and other innovations in clinical learning, providing safest care and safest education, are necessary to enhance educational and clinical experiences. Variation in curriculum is inevitably based on the current climate of clinical environment and moreover, clinical educators will also have to manage student performance assessment. This posed a significant challenge in this transformation of education in response to COVID-19. Although the risk control measures had been in place to safeguard the wellbeing and safety of students, one must also recognize that the current challenging circumstances would have affected the workload and variety of procedures being performed. All non-essential appointments had been deferred with primary focus mainly on emergency medical conditions [6]. Modified clinical performance evaluations were conducted with removal of high-stake summative assessments and reduction of formative competency assessments.

An example was the growing concern from the students on insufficient procedures requiring modification of routine radiography technique. Students were required to perform such procedures in order to fulfil the assessment requirement of the clinical placement. However, the presentation of such procedures varied and could be scarce during the lockdown of the country.

The main approach would be to have timely communication between the HEI and hospital. Clinical educators are now playing a more crucial role in maintaining the integrity of student clinical education experience completion. Reviewing of case logs to ensure that students meet required level of performance with sufficient documentation of direct patient care over their placement period is more vital for close communication between the stakeholders. Educators raised and communicated this concern to the HEI and amendments to assessment were made to the number of case logs required while being conscious of the expectation of nurturing competent, safe, and work-ready radiographers. In addition, HEI utilized simulation training to supplement any gaps in meeting learning outcomes from the reduced number of cases [5]. In partnership, the educators and HEI worked collaboratively to ensure that students met all expectations and competencies for entry level clinical practice in the current healthcare environment prior to graduation [17].

\section{New norms}

As many of us had done in the past few months, students and educators had to adapt to the rapidly changing healthcare and education landscape. During the recently completed student clinical placement, more often than not, educators had to proactively adopt alternative ways of doing things, strive to innovate new ways to deliver clinical training, and seize opportunities to transform the education and education delivery. On the other hand, students had to adapt to the changes in modes of radiography teaching due to COVID-19, while complying with tight health and safety protocol.

Students often deemed temperature monitoring a chore and this was complicated by their clinical placement roster which required some of them to be on shift duties. Despite emphasizing on the need for twice a day temperature records, instances of missing records were not unusual. The educators had to leverage mobile applications to monitor compliance and send reminder 
for daily temperature recording. In addition, peer leaders within the groups were identified and empowered to ensure full compliance by all.

Peer learning has many benefits and may be useful in the clinical learning environment where our student radiographers find themselves overwhelmed and even unsupported [18]. The educators had been using peer learning model for clinical training during pre-COVID clinical placement. However, safe distancing measures had resulted in the reduced educator-student ratio of $1: 1$, disrupting implementation of peer learning in the clinical setting. We were quick to adapt to this new ratio and focused on cultivating closer relationships with our students. In tandem, we were able to provide our students with quicker access to feedback and spent more time to involve them in discussions - supporting them in their clinical learning.

Positive feedbacks were commonly received from pre-COVID tutorials which were facilitated for all students through face-to-face approach. Tutorials covering image analysis, problem-based learning and case-based learning were highly sought after. Recognizing that there would be potential loss in learning opportunities due to the current situation and also the
1:1 clinical education model, the educators were determined to re-introduce COVID era tutorials to complement our present clinical training. Materials from pre-COVID tutorials were currently being reviewed and modified with a plan to re-introduce these tutorials through a teleconference platform at the upcoming student clinical placement. In tandem, educators were getting prepared to deliver these tutorials by gaining exposure to more online teaching so that they were more comfortable with their individual virtual teaching skillset.

\section{Conclusion}

With the resumption of clinical placement, we had to be nimble and needed to rethink our strategy (Fig. 3) to mitigate the crisis while adapting to the "new normal". The global pandemic had inevitably made all of us learn to step outside of our comfort zones, with immense impact on our clinical training and way of life. However, we need to forge forward as the pandemic unfolds with resilience and grit while continuing our efforts to nurture the next generation of professionals and

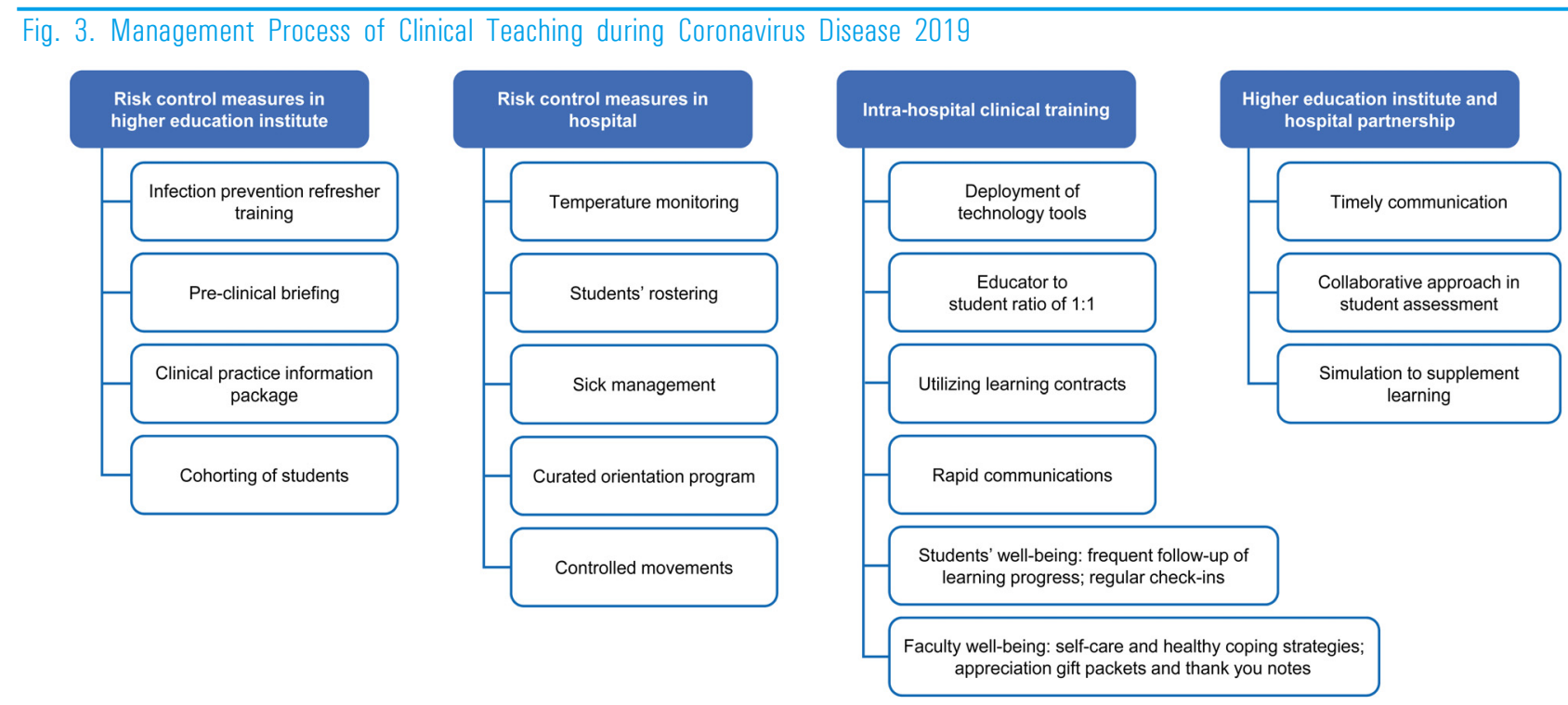


fulfilling our mission as the clinical teacher.

ORCID:

Yi Xiang Tay: https://orcid.org/0000-0001-8322-1500;

Hwei Chuin Chow: https://orcid.org/0000-0003-2059-4144;

Chin Chin Ooi: https://orcid.org/0000-0003-3685-0122

Acknowledgements: None.

Funding: No funding provided for this research.

Conflicts of interest: No potential conflict of interest relevant to this article was reported.

Author contributions: All authors contributed equally in the conception and design of this work, and writing of the manuscript.

\section{References}

1. Singapore Ministry of Health. MOH pandemic readiness and response plan for influenza and other acute respiratory diseases. https://www.moh.gov.sg/docs/librariesprovider5/ diseases-updates/interim-pandemic-plan-public-ver-_april2014.pdf. Published April 2014. Accessed June 2, 2020.

2. Ashokka B, Ong SY, Tay KH, Loh NH, Gee CF, Samarasekera DD. Coordinated responses of academic medical centres to pandemics: sustaining medical education during COVID-19. Med Teach. 2020;42(7): 762-771.

3. Chang D, Xu H, Rebaza A, Sharma L, Dela Cruz CS. Protecting health-care workers from subclinical coronavirus infection. Lancet Respir Med. 2020;8(3):el3.

4. Halbert JA, Jones A, Ramsey LP. Clinical placements for medical students in the time of COVID-19. Med J Aust. 2020;213(2):69.

5. Tay YX, Sng LH, Chow HC, Zainuldin MR. Clinical placements for undergraduate diagnostic radiography students amidst the COVID-19 pandemic in Singapore: preparation, challenges and strategies for safe resumption. J Med Imaging Radiat Sci. 2020:S1939-8654(20) 30224-1.

6. Singapore Ministry of Health. Continuation of essential healthcare services during period of heightened safe distancing measures. https://www.moh.gov.sg/news-highlights /details/continuation-of-essential-healthcare-services-duri ng-period-of-heightened-safe-distancing-measures. Published April 2020. Accessed June 15, 2020.

7. Mathew C, Khee TT. The war on COVID-19 in Singapore: views from the frontline. https://kyspp.nus.edu.sg/gia/ article/the-war-on-covid-19-in-singapore-views-from-the-fr ontline. Published April 2020. Accessed August 25, 2020.

8. American Council of Academic Physical Therapy. Guidance on participation in clinical education experiences in physical therapy education. https://acapt.org/ docs/default-source/public-docs/guidance-on-participation -in-ce-experiences_final3.pdf?sfvrsn $=$ badb8cd8_2\&amp; fvrsn $=$ badb8cd8_2. Published 2020. Accessed August 25, 2020.

9. Gill D, Whitehead C, Wondimagegn D. Challenges to medical education at a time of physical distancing. Lancet. 2020;396(10244):77-79.

10. Singapore Ministry of Manpower. Requirements for safe management measures at the workplace. https://www. mom.gov.sg/covid-19/requirements-for-safe-managementmeasures. Published May 2020. Accessed August 25, 2020.

11. Eng Koon O. The impact of sociocultural influences on the COVID-19 measures: reflections from Singapore. J Pain Symptom Manage. 2020;60(2):e90-e92.

12. Rawle M, Oliver T, Pighills A, Lindsay D. Improving education and supervision of Queensland X-ray operators through video conference technology: a teleradiography pilot project. J Med Radiat Sci. 2017; 64(4):244-250.

13. Rye KJ. Perceived benefits of the use of learning contracts to guide clinical education in respiratory care 
students. Respir Care. 2008;53(11):1475-1481.

14. Ullah R, Amin S. The psychological impact of COVID19 on medical students [Letter]. Psychiatry Res. 2020;288:1 13020.

15. Teo LW, Pang T, Ong YJ, Lai C. Coping with COVID19: perspectives of student radiographers. J Med Imaging Radiat Sci. 2020;51(3):358-360.

16. University of California. Emotional well-being and coping during COVID-19. https://psychiatry.ucsf.edu/ coronavirus/coping. Published 2020. Accessed August 25,
2020.

17. The Quality Assurance Agency for Higher Education. COVID-19: thematic guidance: work-based learning. https://www.qaa.ac.uk/docs/qaa/guidance/covid-19-themat ic-guidance-work-based-learning.pdf?sfvrsn=e3cecd81_1 0. Published 2020. Accessed August 25, 2020.

18. Tai JH, Canny BJ, Haines TP, Molloy EK. Implementing peer learning in clinical education: a framework to address challenges in the "real world". Teach Learn Med. 2017;29(2):162-172. 